\title{
Effectiveness of Dual-Energy Computed Tomography in Providing Information on Pulmonary Perfusion and Vessel Morphology in Chronic Thromboembolic Pulmonary Hypertension
}

Hiroto Shimokawahara, MD; Shun Ijuin, MD; Norihito Nuruki, MD; Masahiro Sonoda, MD

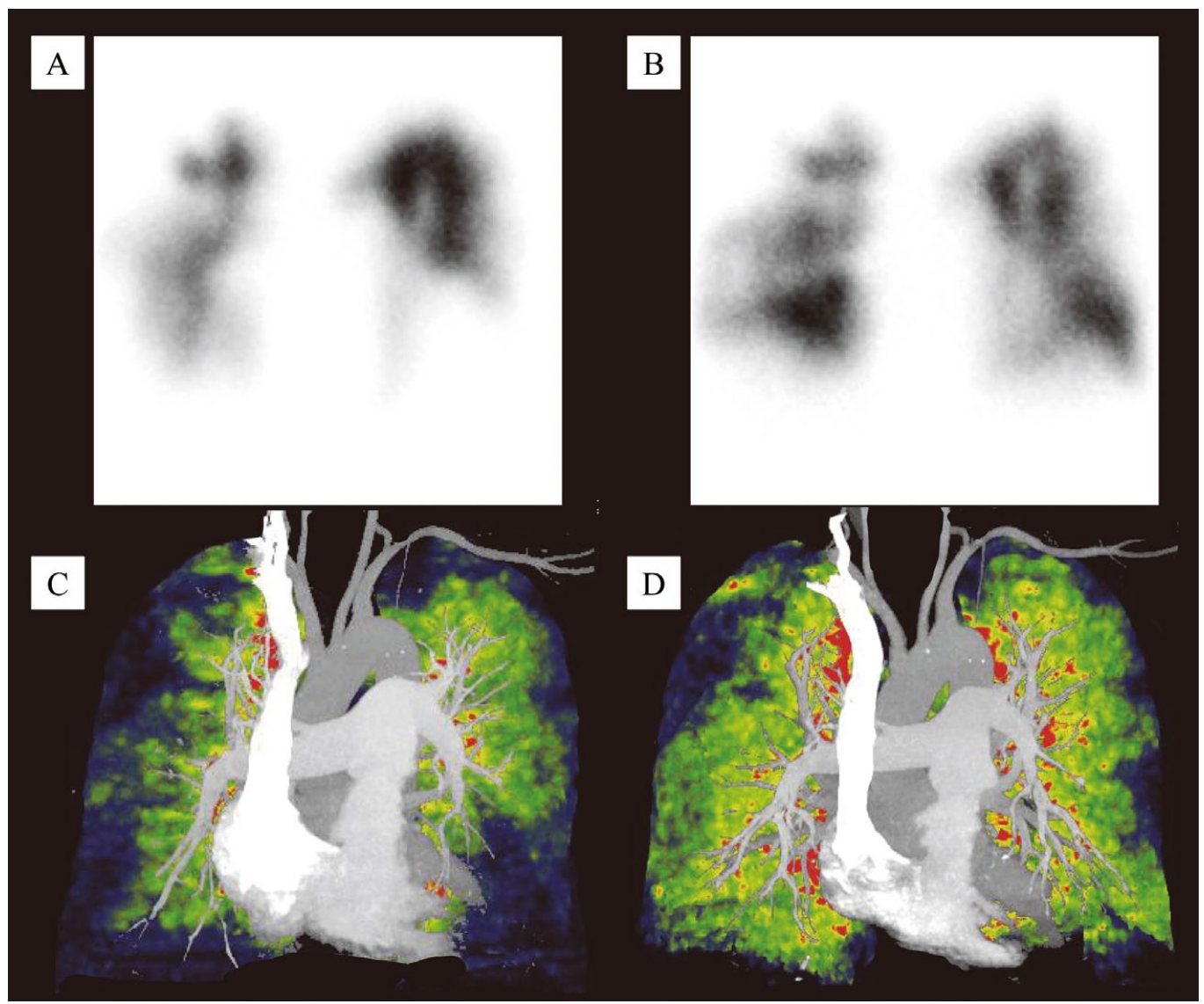

Figure 1. Lung perfusion scintigraphy and dual-energy computed tomography (DECT) lung perfused blood volume CT (PBV-CT) and CT pulmonary angiography (CTPA) before and after balloon pulmonary angioplasty (BPA). (A) Lung perfusion scintigraphy (frontal view) shows multiple wedge-shaped perfusion defects in bilateral lungs before BPA. (B) Multiple perfusion defects on lung perfusion scintigraphy have partially recovered after BPA. (C) DECT (frontal view) CTPA shows multiple stenotic vessels and occluded vessels in the bilateral lung before BPA. Lung PBV-CT shows multiple low-attenuation areas along the stenotic and occluded vessels. (D) DECT, frontal view: multiple low-attenuation areas of the bilateral lung on lung PBV-CT have recovered partially, and the treated pulmonary artery, as well as the pulmonary vein, has enlarged on CTPA after BPA.

Received May 15, 2015; revised manuscript received July 18, 2015; accepted August 3, 2015; released online August 20, 2015 Time for primary review: 26 days

Department of Cardiology, National Hospital Organization Kagoshima Medical Center, Kagoshima, Japan

Mailing address: Hiroto Shimokawahara, MD, Department of Cardiology, National Hospital Organization Kagoshima Medical Center, 8-1

Shiroyama-cho, Kagoshima 892-0853, Japan. E-mail: hiroto.shimokk@gmail.com

ISSN-1346-9843 doi:10.1253/circj.CJ-15-0537

All rights are reserved to the Japanese Circulation Society. For permissions, please e-mail: cj@j-circ.or.jp 


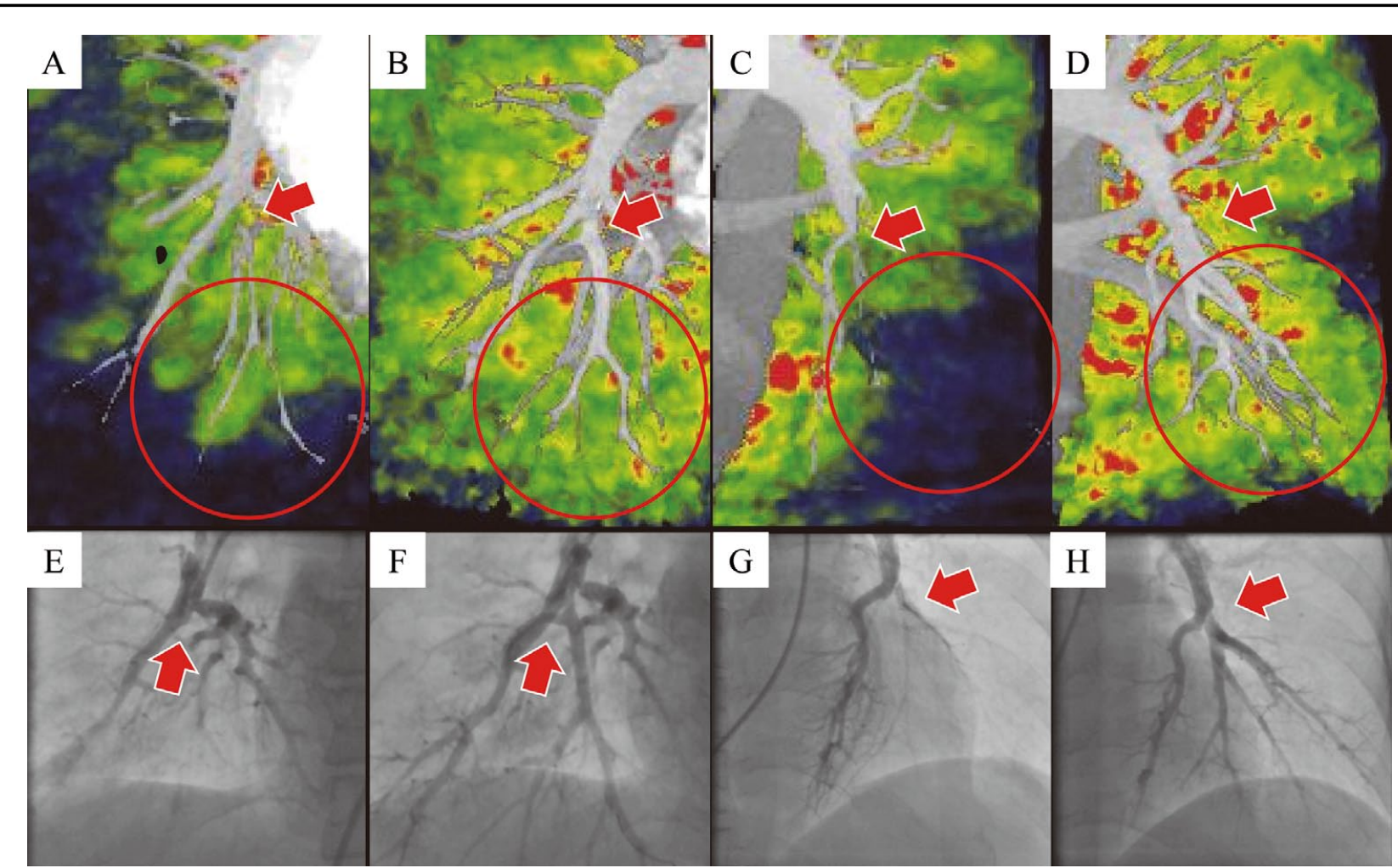

Figure 2. Contrast between partial dual-energy computed tomography (DECT) and selective pulmonary angiography (PAG) before and after balloon pulmonary angioplasty (BPA) in the frontal view. (A) DECT (frontal view) CT pulmonary angiography (CTPA) shows the occluded vessel of the right lateral basal branch (arrow). Lung perfused blood volume CT (PBV-CT) shows a low-attenuation area in the right inferior lobe along the obstructed vessel before BPA. (B) DECT, frontal view: the low-attenuation area of the right inferior lobe has disappeared, and the treated pulmonary vessel has enlarged (arrow) after BPA. (C) DECT (frontal view) CTPA shows occlusion of the left anterior and lateral basal branches (arrow). Lung PBV-CT shows a wedge-shaped low-attenuation area in the left inferior lobe before BPA. (D) DECT, frontal view: the low-attenuation area of the left inferior lobe has disappeared, and obstructed pulmonary vessels have appeared after BPA. (E-H) PAG: (E) avascular area in the right inferior lobe; (F) reperfusion of the right lateral basal branch (arrow) after BPA; (G) nearly completely obstructed vessel in the left inferior lobe (arrow) before BPA; $(\mathbf{H})$ reperfusion of the left lateral basal branch in the left inferior lobe after BPA.

$\mathbf{P}$ ulmonary endarterectomy (PEA) is the most clinically effective treatment for chronic thromboembolic pulmonary hypertension (CTEPH) and remains the only definitive and curative treatment. ${ }^{1}$ Several reports, however, recently demonstrated that balloon pulmonary angioplasty (BPA) improves the clinical status and hemodynamics in inoperable patients with CTEPH. ${ }^{2,3}$ According to the latest guideline, BPA could be considered effective for patients deemed inoperable by an experienced surgeon because of predominant distal disease or comorbidities, or in those with persistent or recurrent pulmonary hypertension after PEA. ${ }^{4}$ Lung perfusion scintigraphy remains a non-invasive and widely used imaging modality for diagnosing CTEPH. Despite its high specificity, this modality seems to lack sensitivity, especially in patients without severe perfusion abnormality. Moreover, neither lung perfusion scintigraphy nor pulmonary angiography (PAG) alone provides sufficient information for selection of target lesions for good therapeutic effect in BPA, because scintigraphy provides only functional information regarding lung perfusion, and PAG provides only morphological information. There has been no definitive modality that evaluates the vessel sufficiently before BPA and can thus assist in selecting target lesions for good therapeutic effect.
We have already reported the value of lung perfused blood volume computed tomography (PBV-CT) using dual-energy CT (DECT) for examination of regional lung perfusion including collateral circulation via the use of differences in absorption of iodine when performing BPA. ${ }^{5}$ DECT can simultaneously evaluate both functional lung perfusion and morphological pulmonary vessel obstructions in a single examination. Given the present results, 3-D lung PBV-CT and CT pulmonary angiography (CTPA) may improve the diagnostic accuracy of CTEPH, including not only the selection of target lesions before BPA, but also detailed evaluation of treatment after BPA.

A 63-year-old woman was referred to hospital with hemoptysis and progressive exertional dyspnea. Lung perfusion scintigraphy showed multiple wedge-shaped perfusion defects in bilateral lung fields (Figure 1A), and she was finally diagnosed as having CTEPH based on selective PAG (Figures 2E,G). Treatment with warfarin and riociguat was started, and her symptoms and exercise capacity improved slightly. Her World Health Organization (WHO) functional class improved from IV to III. Pulmonary vascular resistance decreased from $1,078 \mathrm{dyne} / \mathrm{s} \cdot \mathrm{cn}^{-5}$ to $460 \mathrm{dyne} / \mathrm{s} \cdot \mathrm{cn}^{-5}$, and her cardiac index improved from $2.65 \mathrm{~L} \cdot \mathrm{min}^{-1} \cdot \mathrm{m}^{-2}$ to 
$5.03 \mathrm{~L} \cdot \mathrm{min}^{-1} \cdot \mathrm{m}^{-2}$, but mean pulmonary artery pressure (PAP) decreased from $60 \mathrm{mmHg}$ to only $56 \mathrm{mmHg}$ after drug treatment. She and her family preferred BPA at the present hospital instead of PEA in another hospital because of her history of depression. To obtain good therapeutic effect, target lesions were selected in advance based on the size of the perfusion area on 3-D DECT, as described in the following section. After 46 days of riociguat titration based on the prescribing information, the first session of BPA was performed. Reperfusion of several occluded vessels was achieved, and the lowattenuation area on lung PBV-CT almost completely recovered after a total of 3 sessions of BPA. WHO functional class improved from III to II, and mean PAP decreased from $60 \mathrm{mmHg}$ to $29 \mathrm{mmHg}$. The patient has continued to undergo BPA to achieve a goal of mean PAP $<25 \mathrm{mmHg}$.

Although multiple perfusion defects on lung perfusion scintigraphy were seen to recover after BPA (Figures 1A,B), 3-D DECT enabled more precise and multi-directional evaluation of lung perfusion compared with scintigraphy (Figures 1C,D). Before BPA, lung PBV-CT showed a low-attenuation area in the right inferior lobe, especially in the distal area, along with stenosis of the right lateral basal branch (Figure 2A). On selective PAG, the right lateral basal branch was completely occluded before BPA (Figure 2E), and reperfusion was successful after BPA (Figure 2F). On lung PBV-CT, the lowattenuation area of the right inferior lobe disappeared, and the targeted pulmonary vessel including the pulmonary vein in the perfused area appeared and enlarged after BPA (Figure 2B). Similar findings of 3-D DECT and PAG could be observed in the left inferior lobe (Figures 2C,D,G,H).

Three-dimensional lung PBV-CT and CTPA provides useful information regarding both functional lung perfusion and morphological pulmonary vessel obstructions in a single examination if the problems of radiation exposure and the use of contrast media can be overcome. Previous reports demonstrated that small vessel obstruction observed on lung biopsy during PEA affects postoperative hemodynamics and clinical findings, such as persistent hypoxia. ${ }^{6}$ Much lower attenuation areas on lung PBV-CT may indicate microvasculopathy, which might induce oxygenation disorders in patients with CTEPH. Such information before the procedure would be of great help to operators performing BPA or PEA to ensure successful treatment. In addition, 3-D DECT would be much more useful for detecting minor changes in lung perfusion abnormalities than scintigraphy before and after BPA. It also provides more information non-invasively than PAG regarding lung perfusion and running direction beyond the occluded pulmonary vessels. DECT would be a great help not only in diagnosis, but also in selection of the optimal lesion for effective treatment in patients with CTEPH.

\section{Disclosures}

None.

\section{References}

1. Madani MM, Auger WR, Pretorius V, Sakakibara N, Kerr KM, Kim $\mathrm{NH}$, et al. Pulmonary endarterectomy: Recent changes in a single institution's experience of more than 2,700 patients. Ann Thorac Surg 2012; 94: 97-103.

2. Sugimura K, Fukumoto Y, Satoh K, Nochioka K, Miura Y, Aoki T, et al. Percutaneous transluminal pulmonary angioplasty markedly improves pulmonary hemodynamics and long-term prognosis in patients with chronic thromboembolic pulmonary hypertension. Circ J 2012; 76: 485-488.

3. Tatebe S, Fukumoto Y, Sugimura K, Miura Y, Nochioka K, Aoki T, et al. Optical coherence tomography is superior to intravascular ultrasound for diagnosis of distal-type chronic thromboembolic pulmonary hypertension. Circ J 2013; 77: 1081-1083.

4. Schweikert B, Pittrow D, Vizza CD, Pepke-Zaba J, Hoeper MM, Gabriel A, et al. Chronic thromboembolic pulmonary hypertension. Lancet Respir Med 2014; 2: 573-582.

5. Shimokawahara H, Ijuuin S, Sonoda K, Sai E, Yamashita E, Tanoue $\mathrm{K}$, et al. The value of lung perfused blood volume computed tomography in selecting the target lesions for the effective treatment of chronic thromboembolic pulmonary hypertension. Circulation 2014; 130: $448-449$.

6. Yamaki S, Ando M, Fukumoto Y, Higuchi Y, Kaneko K, Maeda K, et al. Histopathological examination by lung biopsy for the evaluation of operability and postoperative prognosis in patients with chronic thromboembolic pulmonary hypertension. Circ J 2014; 78: $476-482$. 